the relevant information. Developing a well-formulated research question is one of the challenging tasks an occupational health professional encounters when planning a public health intervention.

The detailed specification of the question will guide many aspects of the research including the formulation of the search strategy. The structure of the search strategy should be based on a clearly defined research question. The World Health Organisation suggests the PICO model to help defining the question. This acronym stands for Population (P), Intervention (I), Comparison (C), and Outcome (O). You cannot simply use the PICO terms to search in PubMed - the free search engine maintained by the US National Library of Medicine. You have to translate them into search terms. There are two types of search terms: the Medical Subject Headings (MeSH) terms and the 'free text words' (ie. non-MeSH terms). First, you have to define the $\mathrm{MeSH}$ terms from the PubMed thesaurus to produce highly specific searches. Then, it is recommended to include 'free-text words' considering that these search strategies are usually more sensitive than those containing $\mathrm{MeSH}$ terms only. When using non-MeSH terms, it is important to check for synonyms of the selected non-MeSH terms (eg. tennis elbow and epicondylitis), taking into account differences between British and American spelling and vocabulary.

In addition, you can take advantage of ready-to-use search filters for prompt identification of pertinent literature available in PubMed.

\section{C WHAT STUDY DESIGNS CAN BE UTILISED TO EVALUATE THE EFFECTIVENESS OF INTERVENTIONS?}

JH Ruotsalainen. Cochrane Work, Finnish Institute of Occupational Health, Kuopio, Finland

\subsection{6/oemed-2018-ICOHabstracts.452}

Introduction When evaluating the effectiveness of an intervention it is imperative that one uses the research design with the smallest possible risk of bias (i.e. highest methodological quality).

Methods During this talk I will present a range of study designs that can be used to conduct assessments of intervention effectiveness. These range from the established gold standard for clinical interventions, the randomised controlled trial (RCT), to various non-randomised designs, time-series and case-control studies.

Result It will hopefully become apparent that a lot of the criticism against RCTs being too difficult in occupational health settings is actually often based on misconceptions. At the same time, it is also true that the RCT is not a one size fits all solution, i.e. the only study design that can yield informative results. It is good to understand that there is a gradient from low inherent risk bias methods, such as the RCT, that yield the most reliable results to increasing risk of bias and less reliable results when, for example, one relaxes the demands for removing various sources of confounding.

Discussion Conducting an intervention effectiveness study requires considerable investment in terms of time, money and resources. At worst all this goes to waste by planning, conducting and reporting studies that are either uninformative, unreliable or both.
$1652 d$

PREVENTIVE OCCUPATIONAL HEALTH INTERVENTIONS: SOME WORKPLACE EXAMPLES

HFvan der Molen, JK Sluiter, MHWFrings-Dresen. Academic Medical Centre, University of Amsterdam, Department: Coronel Institute of Occupational Health, Amsterdam Public Health research institute, Amsterdam, The Netherlands

\subsection{6/oemed-2018-ICOHabstracts.453}

Three examples of evaluating Dutch workplace interventions will be presented. This is input for discussing the essential elements in evaluations of interventions at workplaces on outcomes for work-related diseases or injuries.

i. Needle stick injuries (NSIs) are frequently reported as occupational injuries among health care workers. The objective of a cluster randomised trial was to evaluate whether the number of NSIs decreased among health care workers at risk $(n=796)$ after introduction of injection needles with safety devices in combination with an interactive workshop in an academic hospital. This combined intervention led to the highest reduction in the number of self-reported NSIs compared to a workshop alone or no intervention.

ii. Low back complaints are frequently reported as workrelated diseases among bricklayers. The objective of a within-subject controlled intervention study was to evaluate whether work related risk factors and discomfort of the low back decreased among bricklayers $(n=10)$ after introducing a scaffolding console to adjust the working height of the storage of materials in a construction company. Working with a scaffolding console resulted in a significant reduction of the frequency and duration of trunk flexion $79 \%$ and $52 \%$ respectively, and as well as discomfort of the lower back compared with bricks set out on the ground floor.

iii. Occupational risks affect the health of construction workers. The objective of a non-randomised controlled trial was to evaluate whether a job-specific workers' health surveillance improved the preventive actions of construction workers $(n=374)$ following occupational physicians recommendations. In total $73 \%$ of the workers undertook job-specific actions versus 59\% in the control group, while occupational physicians provided more job-specific recommendations in the intervention group.

Risk assessment, context related interventions and compliance to interventions are essential elements before and alongside the evaluation of real world preventive interventions.

\section{EFFECTIVENESS OF PERIODIC HEALTH EXAMINATION IN EUROPE AND ASIA}

${ }^{1}$ Seong-Kyu Kang*, '2Stefano Mattioli*. ' Gachon University Gil Medical Centre, Incheon, Republic of Korea; ${ }^{2}$ University of Bologna, Bologna, Italy

\subsection{6/oemed-2018-ICOHabstracts.454}

Aim of special session The session will give the audience the current status, effectiveness, challenge, and new perspectives of periodic health (medical) examination in Europe and Asia, which is widely performed in many countries as one of tools of occupational health service for all workers. 